\title{
Shedding of OXA-181 carbapenemase-producing Escherichia coli from companion animals after hospitalisation in Switzerland: an outbreak in 2018
}

Aurélien Nigg ${ }^{1}$, Michael Brilhante ${ }^{1,2}$, Valentina Dazio ${ }^{3}$, Mathieu Clément ${ }^{2,5}$, Alexandra Collaud ${ }^{1}$, Stefanie Gobeli Brawand ${ }^{1}$,

Barbara Willi ${ }^{4}$, Andrea Endimiani ${ }^{5}$, Simone Schuller ${ }^{3}$, Vincent Perreten ${ }^{1}$

1. Institute of Veterinary Bacteriology, Bern, University of Bern

2. Graduate School of Cellular and Biomedical Sciences, Bern, University of Bern

3. Department of Clinical Veterinary Medicine, Bern, University of Bern

4. Clinic for Small Animal Internal Medicine, University of Zurich, Zurich, Switzerland

5. Institute for Infectious Diseases, University of Bern, Bern, Switzerland

Correspondence: Vincent Perreten(vincent.perreten@vetsuisse.unibe.ch)

Citation style for this article: Nigg Aurélien, Brilhante Michael, Dazio Valentina, Clément Mathieu, Collaud Alexandra, Gobeli Brawand Stefanie, Willi Barbara, Endimiani Andrea, Schuller
Simone, Perreten Vincent. Shedding of OXA-181 carbapenemase-producing Escherichia coli from companion animals after hospitalisation in Switzerland: an outbreak in 2018. Euro Surveill. 2019;24(39):pii=1900071. https://doi.org/10.2807/1560-7917.ES.2019.24.39.1900071

Background: Carbapenem-resistant Enterobacteriaceae pose a serious threat to public health worldwide, and the role of companion animals as a reservoir is still unclear. Aims: This 4-month prospective observational study evaluated carriage of carbapenem-resistant Enterobacteriaceae at admission and after hospitalisation in a large referral hospital for companion animals in Switzerland. Methods: Rectal swabs of dogs and cats expected to be hospitalised for at least $48 \mathrm{~h}$ were taken from May to August 2018 and analysed for the presence of carbapenem-resistant Enterobacteriaceae using selective agar plates. Resistant isolates were further characterised analysing whole genome sequences for resistance gene and plasmid identification, and ad hoc core genome multilocus sequence typing. Results: This study revealed nosocomial acquisition of Escherichia coli harbouring the carbapenemase gene bla ${ }_{\text {oxA-181 }}$, the $\mathrm{pAmpC}$ cephalosporinase gene $b_{\mathrm{CMY}-42}$ as well as quinolone resistance associated with qnrS1 and mutations in the topoisomerases II (GyrA) and IV (ParC). The bla ${ }_{\text {oxA-181 }}$ and $q n r S_{1}$ genes were identified on a $51 \mathrm{~kb}$ IncX3 plasmid and bla ${ }_{\mathrm{CMY}-42}$ on a $47 \mathrm{~kb}$ Incl1 plasmid. All isolates belonged to sequence type ST410 and were genetically highly related. This $E$. coli clone was detected in 17 of 100 dogs and four of 34 cats after hospitalisation (21.6\%), only one of the tested animals having tested positive at admission ( $0.75 \%)$. Two positive animals were still carriers 4 months after hospital discharge, but were negative after 6 months. Conclusions: Companion animals may acquire carbapenemaseproducing $E$. coli during hospitalisation, posing the risk of further dissemination to the animal and human population and to the environment.

\section{Introduction}

Companion animals nowadays not only share the owner's home environment but also benefit from intensive veterinary care in case of serious illness. Veterinary referral hospital environments are similar to human hospitals in that animal patients face similar risks of developing nosocomial infections.

Bacterial infections in dogs and cats are frequently treated with critically important antimicrobials such as fluoroquinolones or cephalosporins. In very rare cases, even the last resort antibiotics of human medicine such as carbapenems may be used in companion animals in Switzerland to treat infections refractory to any other standard antimicrobial used in veterinary medicine (Ordinance on Veterinary Medicinal Products, SR 812.212.27, Art. 6).

Such treatments pose the risk of selecting resistance to these classes of antibiotics in Enterobacteriaceae through the acquisition of plasmid-mediated cephalosporinase genes (e.g. bla стх-м $_{\text {, bla }}$ сму ) and fluoroquinolones resistance genes (e.g. $q n r, a a c-(6)-l b-c r)$, but also through chromosomal mutations in the promotor region of the AmpC $\beta$-lactamase and in the quinolone resistance-determining region (QRDR) of the topoisomerases II (GyrA) and IV (ParC) [1,2]. Resistances to carbapenems have also emerged in Escherichia coli and Klebsiella pneumoniae [3]. Three main groups of carbapenemases are referenced according to the Ambler classification: class A (e.g. KPC), class B (IMP, VIM, NDM) and class D (e.g. OXA) [4]. Among the OXA carbapenemases, OXA-48-like ones have been described as phantom carbapenemases since they 


\section{FIGURE 1}

Temporal acquisition and carriage of carbapenemase-producing Escherichia coli ST410, Switzerland, May-August 2018 $(\mathrm{n}=24)$

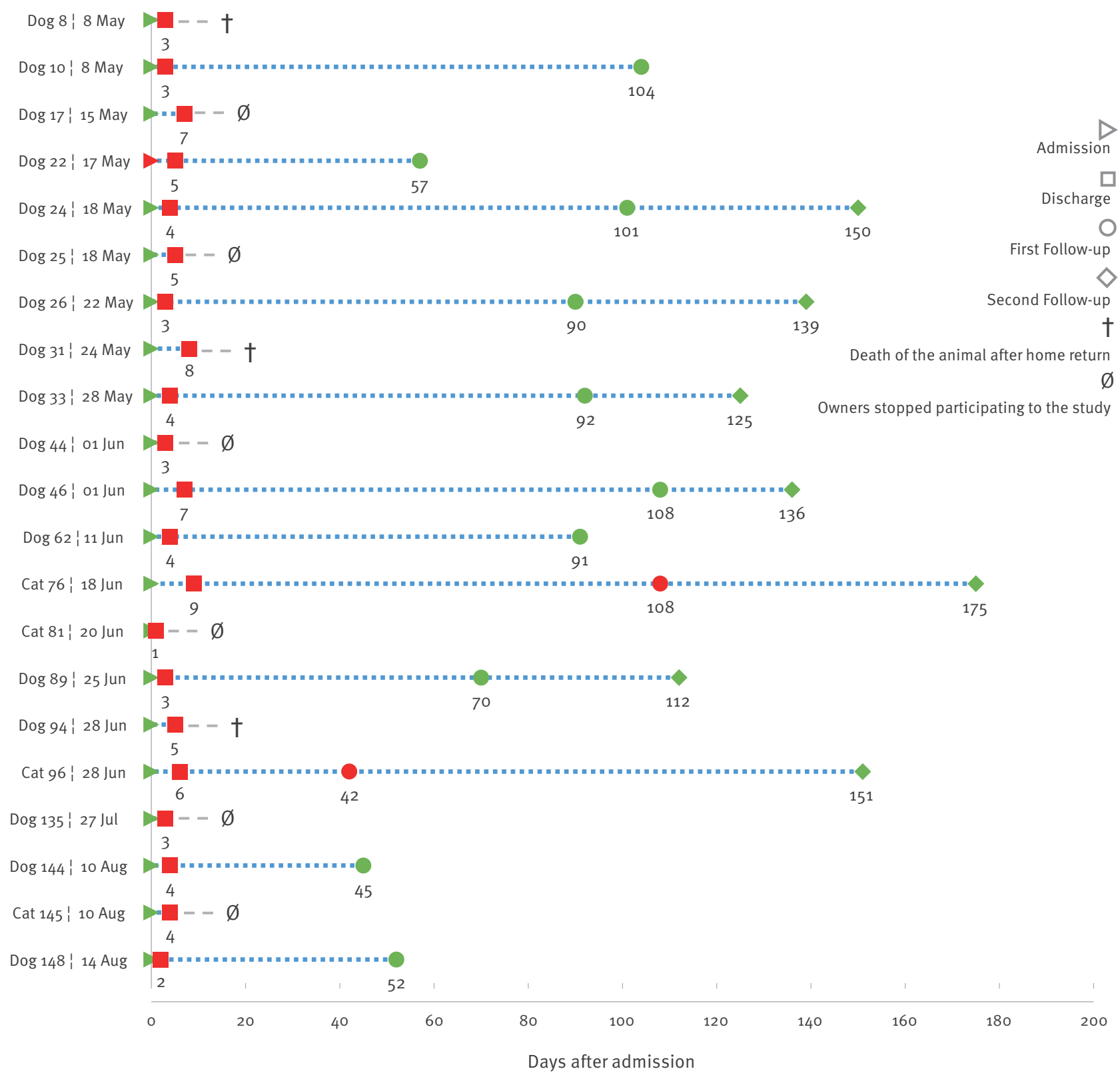

Results as detected at admission and discharge to a companion animal clinic, as well as once or twice within 175 days after hospitalisation.

Shapes in green: test negative for CP E. coli carriage; shapes in red: test positive for CP E. coli carriage. Blue dotted line: time line of screened animals; grey dashed line: animals which were not resampled.

may be difficult to detect using phenotypic susceptibility testing [5].

Until now, animals were considered to play a minor role in the dissemination of OXA-48-like carbapenemases [6], but reports of sporadic infections in dogs caused by carbapenemase-producing (CP) Enterobacteriaceae in Europe [7,8], in the United States (US) [9] and in
Northern Africa [10] suggest that companion animals may represent an unsuspected reservoir of carbapenem-resistant bacteria. Recently, hospitalised companion animals in Italy were found to acquire carbapenem-resistant bacteria such as Acinetobacter spp. [11]. Nonetheless, very little is known about carriage and dynamics of CPE in dogs and cats before and after hospitalisation. 
TABLE 1

Demographic, hospitalisation and antimicrobial treatment details of dogs and cats positive for OXA181producing Escherichia coli ST410, Switzerland, May-August 2018 ( $\mathrm{n}=21)$

\begin{tabular}{|c|c|c|}
\hline Parameter & Dogs $(n=17)$ & Cats $(n=4)$ \\
\hline Age median in years (IQR) & $7.0(4.0-9.0)$ & $4.5(2.3-10.5)$ \\
\hline Weight median in kg (IQR) & $16.6(7.8-34)$ & $4.2(3.0-5.7)$ \\
\hline \multicolumn{3}{|l|}{ Sex } \\
\hline Female (entire/neutered) & $9(5 / 4)$ & $2(0 / 2)$ \\
\hline Male (entire/neutered) & $8(6 / 2)$ & $2(1 / 1)$ \\
\hline \multicolumn{3}{|l|}{ Diagnoses } \\
\hline Gastrointestinal disease & 7 & 1 \\
\hline Neurological disease & 5 & 0 \\
\hline Urinary tract disease & 2 & 2 \\
\hline Other & 3 & 1 \\
\hline Hospitalisation days; median (IQR) & $4(3-5)$ & $5(1.8-8.3)$ \\
\hline ICU days; median (IQR) & $3(1-5)$ & $2.3(1.5-8.6)$ \\
\hline \multicolumn{3}{|l|}{ Antimicrobial pre-treatment ${ }^{\mathrm{a}}$} \\
\hline Yes & 4 & 1 \\
\hline No & 11 & 3 \\
\hline Unknown & 2 & 0 \\
\hline \multicolumn{3}{|c|}{ Antimicrobial treatment during hospitalisation } \\
\hline Yes & 14 & 3 \\
\hline No & 3 & 1 \\
\hline \multicolumn{3}{|c|}{ Antimicrobials used during hospitalisation ${ }^{b}$} \\
\hline Ampicillin/sulbactam & 9 & 3 \\
\hline Cefazolin & 1 & 1 \\
\hline Clindamycin & 2 & 0 \\
\hline Doxycycline & 1 & 0 \\
\hline Enrofloxacin & 1 & 0 \\
\hline Metronidazole & 1 & 0 \\
\hline Sulfamethoxazole/trimethoprim & 1 & o \\
\hline Amoxicillin/clavulanic acid & 1 & 0 \\
\hline
\end{tabular}

ICU: intensive care unit; IQR: interquartile range.

${ }^{a}$ Antimicrobials used for pre-treatments (i.e. prior to presentation/referral) were amoxicillin/clavulanic acid $(n=2)$, amoxicillin $(n=1)$, enrofloxacin $(n=1)$ and metronidazole $(n=1)$.

${ }^{b}$ Includes mono- and combination therapy.

Here, we characterised by whole genome sequencing (WGS) CP E. coli isolated from cats and dogs at admission and after hospitalisation to a large veterinary referral hospital in Switzerland, identifying their antimicrobial resistance genes (ARG) and mobile genetic elements (MGE) as well as the phylogenetic relationship among the isolates.

\section{Methods}

\section{Study design and sampling}

This study was part of a larger prospective study which included three large veterinary referral clinics and two smaller veterinary practices in Switzerland. The present prospective observational study was performed at a university referral hospital with a $24 / 7$ emergency service. The hospital provides advanced medical care for 6,000 dogs and cats annually. Dogs and cats were included irrespective of their underlying problems and previous treatments if they were expected to be hospitalised for at least $48 \mathrm{~h}$ and the owner gave informed consent. All animals presented here were recruited via the emergency service of the hospital. During the enrolment period of 4 months (1 May-31 August 2018), ca 2,000 animals were treated at the hospital and 134 of them fulfilled the study criteria and were screened.

Rectal swabs were collected in awake animals at admission to the hospital and at discharge, using polyurethane foam culture swabs with Ames transport medium (BBL CultureSwabs). If available, animals positive at discharge were retested once or twice within 36 to 166 days after their discharge of the clinic.

\section{Isolation and identification of the strains}

Swabs were placed into $5 \mathrm{~mL}$ of Luria-Bertani (LB) broth for overnight enrichment at $37^{\circ} \mathrm{C}$. A loopful of the culture was streaked onto CHROMID OXA-48 


\section{FIGURE 2}

Phylogenetic neighbour-joining tree of all carbapenemase-producing Escherichia coli of ST410 isolated from animals hospitalised in a companion animal clinic $(n=24)$ and others available in GenBank $(n=7)$, Switzerland, May-August 2018

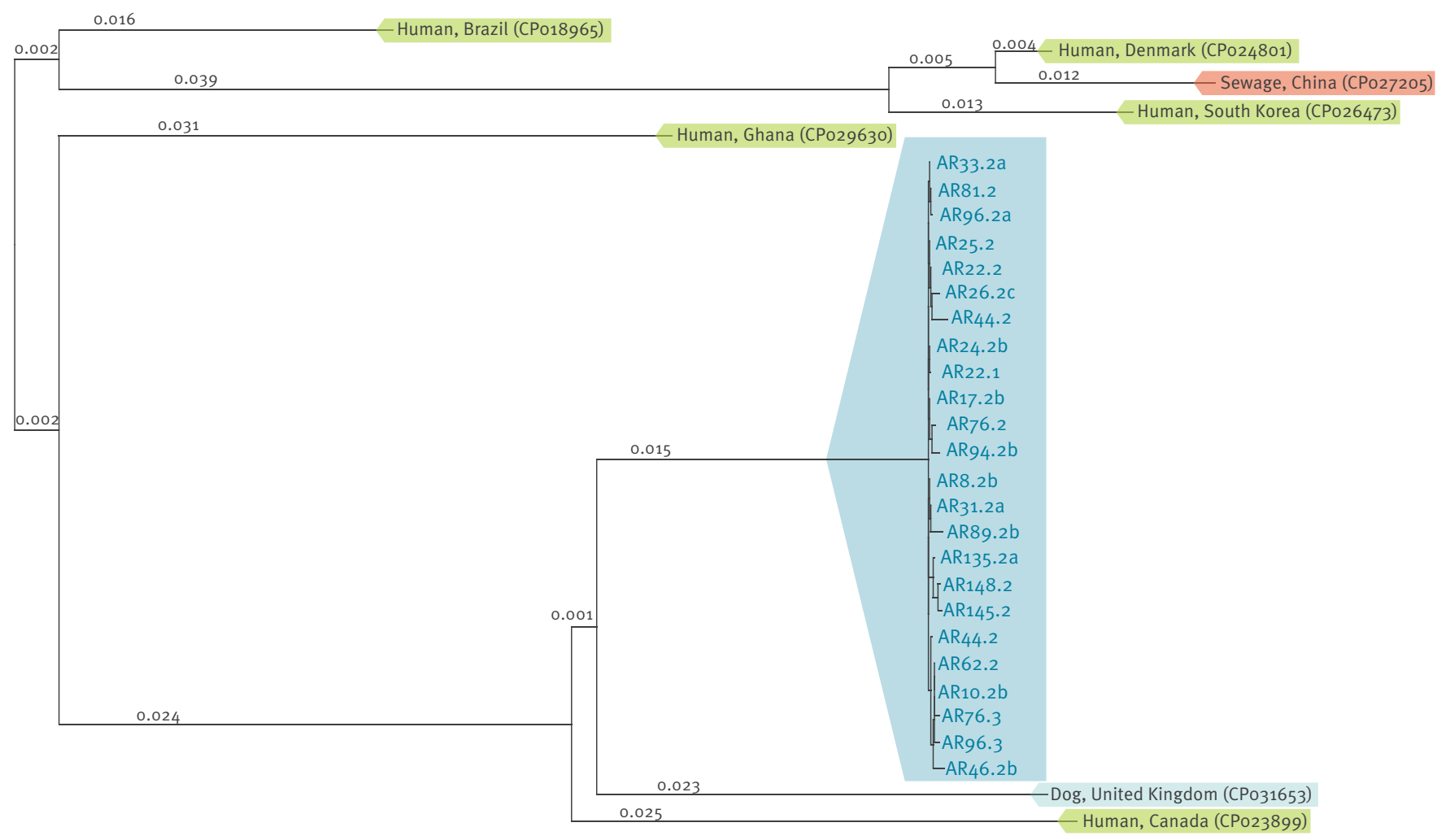

0.01

The tree was generated and drawn using SeqSphere+(v. 401, Rindom GmbH, Münster, Germany) for the comparison of 3,778 genes from the core genome (cgMLST) of the genomic sequences.

Blue: isolates from animals; green: isolates from humans; orange: isolates from the environment.

E. coli isolates used for the comparison were carrying the following resistance genes to $\beta$-lactams, cephalosporins and carbapenems:

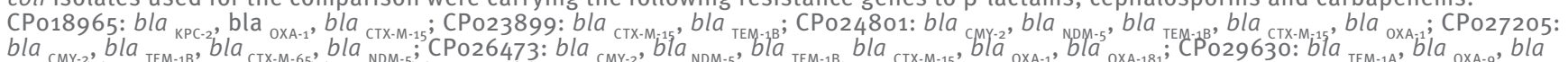

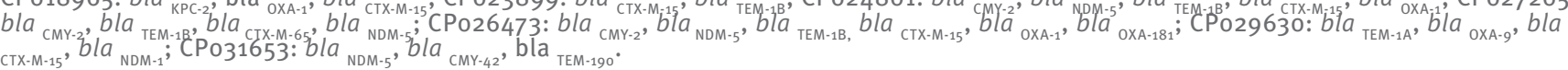

and CHROMID CARBA (Biomérieux SA, Marcy-l'Étoile, France) selective plates, which were incubated at $37^{\circ} \mathrm{C}$ for $24 \mathrm{~h}$ under aerobic conditions.

Colonies were sub-cultivated onto trypton soy agar plates containing $5 \%$ sheep blood (TSA-S) (Becton and Dickinson Company, Franklin Lakes, US) and identified to the species level by matrix-assisted laser desorption/ionisation time-of-flight mass spectrometry (MALDI-TOF MS) (Bruker Daltonics GmbH, Bremen, Germany).

\section{Biochemical and molecular tests}

Carbapenemase production was detected using the Blue-Carba test [12]. Before WGS, carbapenemase-producing isolates were tested for the presence of bla 48-like genes by PCR [5].

\section{Antimicrobial susceptibility testing}

Minimal inhibitory concentrations (MIC) of 16 antimicrobials (ampicillin, cefepime, cefotaxime, ceftazidime, chloramphenicol, ciprofloxacin, colistin, ertapenem, gentamicin, imipenem, meropenem, nalidixic acid, sulfamethoxazole, tetracycline, tigecycline and trimethoprim) were determined by broth microdilution using Sensititre EUVSEC and EUVSEC2 plates (Thermo Fisher Scientific, Waltham, US) and following the guidelines from the European Committee on Antimicrobial Susceptibility Testing (EUCAST) [13]. MIC results were interpreted using the EUCAST criteria, except for nalidixic acid, sulfamethoxazole and tetracycline, for which criteria from the Clinical and Laboratory Standards Institute (CLSI) were used [14]. 
TABLE 2

Minimum inhibitory concentrations of 16 antibiotics for carbapenemase-producing Escherichia coli isolates of ST410 from cats and dogs, Switzerland, May-August $2018(\mathrm{n}=24)$

\begin{tabular}{|c|c|c|c|c|c|c|c|c|c|c|c|c|c|c|c|c|c|c|}
\hline \multicolumn{3}{|c|}{$\begin{array}{l}\text { MIC of antibiotics in } \mu \mathrm{g} / \mathrm{mL}^{\mathrm{a}} \text { and } \\
\text { resistance breakpoints }{ }^{\mathrm{b}} \text { in } \\
\text { parentheses }\end{array}$} & \multirow[t]{2}{*}{$\begin{array}{l}\text { AMP } \\
(>8)\end{array}$} & \multirow[t]{2}{*}{$\begin{array}{l}\mathrm{CHL} \\
(>8)\end{array}$} & \multirow[t]{2}{*}{$\begin{array}{c}\text { CIP } \\
(>0.5)\end{array}$} & \multirow[t]{2}{*}{$\begin{array}{l}\mathrm{COL} \\
(>2)\end{array}$} & \multirow[t]{2}{*}{$\begin{array}{c}\text { ETP } \\
(>0.5)\end{array}$} & \multirow[t]{2}{*}{$\begin{array}{l}\text { FEP } \\
(>4)\end{array}$} & \multirow[t]{2}{*}{$\begin{array}{l}\text { FOT } \\
(>2)\end{array}$} & \multirow[t]{2}{*}{$\begin{array}{l}\text { GEN } \\
(>4)\end{array}$} & \multirow[t]{2}{*}{$\begin{array}{l}\mid \mathrm{MI} \\
(>4)\end{array}$} & \multirow[t]{2}{*}{$\begin{array}{l}\text { MERO } \\
(>8)\end{array}$} & \multirow[t]{2}{*}{$\begin{array}{l}\text { NAL } \\
(\geq 32)\end{array}$} & \multirow[t]{2}{*}{$\begin{array}{c}\text { SMX } \\
(\geq 512)\end{array}$} & \multirow[t]{2}{*}{$\begin{array}{l}\text { TAZ } \\
(>4)\end{array}$} & \multirow[t]{2}{*}{$\begin{array}{c}\text { TET } \\
(\geq 16)\end{array}$} & \multirow[t]{2}{*}{$\begin{array}{l}\text { TGC } \\
(>2)\end{array}$} & \multirow[t]{2}{*}{$\begin{array}{l}\text { TMP } \\
(>4)\end{array}$} \\
\hline Strain & Animal & $\begin{array}{l}\text { Time point } \\
\text { of isolation }\end{array}$ & & & & & & & & & & & & & & & & \\
\hline AR8.2b & Dog 8 & Discharge & $>64$ & $\leq 8$ & 18 & $\leq 1$ & $>2$ & 8 & 24 & $\leq 0.5$ & 1 & 0.5 & $>128$ & 16 & $>128$ & 4 & 0.5 & 0.5 \\
\hline$A R 10.2 b$ & Dog 10 & Discharge & $>64$ & $\leq 8$ & 18 & $\leq 1$ & 12 & 8 & 24 & $\leq 0.5$ & 0.5 & 0.5 & $>128$ & 32 & $>128$ & 4 & $\leq 0.25$ & 1 \\
\hline$A R 17.2 b$ & Dog 17 & Discharge & $>64$ & $\leq 8$ & $>8$ & $\leq 1$ & $>2$ & 8 & 24 & $\leq 0.5$ & 0.5 & 0.5 & $>128$ & 16 & $>128$ & 4 & 0.5 & 0.5 \\
\hline AR22.1 & Dog 22 & Admission & $>64$ & $\leq 8$ & 18 & $\leq 1$ & 12 & 8 & 24 & $\leq 0.5$ & 1 & 0.5 & $>128$ & 16 & $>128$ & 4 & 0.5 & 0.5 \\
\hline$A R 22.2$ & Dog 22 & Discharge & $>64$ & $\leq 8$ & 18 & $\leq 1$ & $>2$ & 16 & 24 & 1 & 0.5 & 0.5 & $>128$ & 16 & $>128$ & 4 & 0.5 & 0.5 \\
\hline$A R 24.2 b$ & Dog 24 & Discharge & $>64$ & $\leq 8$ & 18 & $\leq 1$ & 2 & 8 & 14 & $\leq 0.5$ & 0.5 & 0.25 & $>128$ & 32 & $>128$ & 4 & $\leq 0.25$ & 2 \\
\hline$A R 25.2$ & Dog 25 & Discharge & $>64$ & $\leq 8$ & 28 & $\leq 1$ & 2 & 8 & 24 & $\leq 0.5$ & 0.5 & 0.5 & $>128$ & 16 & $>128$ & 8 & 0.5 & 1 \\
\hline$A R 26.2 C$ & Dog 26 & Discharge & $>64$ & $\leq 8$ & 18 & $\leq 1$ & 12 & 8 & 24 & $\leq 0.5$ & 1 & 0.5 & $>128$ & 16 & $>128$ & 4 & 0.5 & 1 \\
\hline$A_{31.2 a}$ & Dog 31 & Discharge & $>64$ & $\leq 8$ & $>8$ & $\leq 1$ & $>2$ & 8 & $2 / 4$ & $\leq 0.5$ & 0.5 & 0.5 & $>128$ & 16 & $>128$ & 4 & 0.5 & 1 \\
\hline$A R 33.2 a$ & Dog 33 & Discharge & $>64$ & $\leq 8$ & 18 & $\leq 1$ & 2 & 8 & 74 & $\leq 0.5$ & 0.5 & 0.5 & $>128$ & 16 & $>128$ & 4 & 0.5 & 1 \\
\hline AR44.2 & Dog 44 & Discharge & $>64$ & $\leq 8$ & 18 & $\leq 1$ & 2 & 8 & $>4$ & $\leq 0.5$ & 0.5 & 0.5 & $>128$ & 16 & $>128$ & 4 & 0.5 & 0.5 \\
\hline$A R 46.2 b$ & Dog 46 & Discharge & $>64$ & $\leq 8$ & 18 & $\leq 1$ & 2 & 8 & 24 & $\leq 0.5$ & 0.5 & 0.25 & $>128$ & 16 & $>128$ & 4 & $\leq 0.25$ & 1 \\
\hline AR62.2 & Dog 62 & Discharge & $>64$ & $\leq 8$ & 18 & $\leq 1$ & 2 & 8 & 14 & $\leq 0.5$ & 0.5 & 0.5 & $>128$ & 16 & $>128$ & 4 & 0.5 & 1 \\
\hline AR76.2 & Cat 76 & Discharge & $>64$ & $\leq 8$ & 18 & $\leq 1$ & 2 & 8 & 24 & $\leq 0.5$ & 0.5 & 0.5 & $>128$ & 16 & $>128$ & 4 & 0.5 & 1 \\
\hline AR81.2 & Cat 81 & Discharge & $>64$ & $\leq 8$ & 18 & $\leq 1$ & 12 & 8 & 14 & $\leq 0.5$ & 0.5 & 0.5 & $>128$ & 16 & $>128$ & 4 & 0.5 & 1 \\
\hline AR89.2b & Dog 89 & Discharge & $>64$ & $\leq 8$ & 18 & $\leq 1$ & 2 & $2^{c}$ & 24 & $\leq 0.5$ & 0.5 & 0.5 & $>128$ & 16 & $2^{c}$ & 4 & 0.5 & 0.5 \\
\hline AR94.2b & Dog 94 & Discharge & $>64$ & $\leq 8$ & 18 & $\leq 1$ & 12 & 8 & 14 & $\leq 0.5$ & 1 & 0.5 & $>128$ & $\leq 8$ & $>128$ & 4 & $\leq 0.25$ & 0.5 \\
\hline AR96.2a & Cat 96 & Discharge & $>64$ & $\leq 8$ & 28 & $\leq 1$ & 2 & 8 & $2 / 4$ & 1 & 0.5 & 0.5 & $>128$ & 16 & $>128$ & 8 & 0.5 & 0.5 \\
\hline AR135.2a & Dog 135 & Discharge & $>64$ & $\leq 8$ & 28 & $\leq 1$ & 12 & 16 & 24 & $\leq 0.5$ & 0.5 & 1 & $>128$ & 16 & $>128$ & 8 & 0.5 & 0.5 \\
\hline AR144.2 & Dog 144 & Discharge & $>64$ & $\leq 8$ & 18 & $\leq 1$ & 2 & $2^{c}$ & 24 & $\leq 0.5$ & 0.5 & 0.25 & $>128$ & 16 & 1 & $>64$ & $\leq 0.25$ & 1 \\
\hline AR145.2 & Cat 145 & Discharge & $>64$ & $\leq 8$ & 18 & $\leq 1$ & 2 & 8 & 24 & $\leq 0.5$ & 0.5 & 0.25 & $>128$ & 16 & $>128$ & 4 & $\leq 0.25$ & 1 \\
\hline AR148.2 & Dog 148 & Discharge & $>64$ & $\leq 8$ & 28 & $\leq 1$ & 2 & 8 & 24 & $\leq 0.5$ & 0.5 & 0.5 & $>128$ & 16 & $>128$ & 4 & $\leq 0.25$ & 1 \\
\hline AR76.3 & Cat 76 & Follow-up & $>64$ & $\leq 8$ & 18 & $\leq 1$ & 2 & 8 & 24 & $\leq 0.5$ & 0.25 & 0.25 & $>128$ & 16 & $>128$ & 4 & $\leq 0.25$ & 1 \\
\hline AR96.3a & Cat 96 & Follow-up & $>64$ & $\leq 8$ & 18 & $\leq 1$ & 2 & 8 & 14 & $\leq 0.5$ & 0.5 & 0.25 & $>128$ & 16 & $>128$ & 4 & $\leq 0.25$ & 1 \\
\hline \multicolumn{3}{|c|}{ Number of non-susceptible strains } & 24 & 0 & 24 & 0 & 24 & 24 & 24 & 0 & 0 & 0 & 24 & 0 & 23 & 1 & 0 & 0 \\
\hline
\end{tabular}

AMP: ampicillin; CHL: chloramphenicol; CIP: ciprofloxacin; COL: colistin; ETP: ertapenem; FEP: cefepime; FOT: cefotaxime; GEN: gentamicin; IMI: imipenem; MERO: meropenem; MIC: minimum inhibitory concentration; NAL: nalidixic acid; SMX: sulfamethoxazole; TAZ: ceftazidime; TET: tetracycline; TGC: tigecycline; TMP: trimethoprim.

${ }^{a}$ MICs highlighted in bold indicate resistance.

b The resistance breakpoints presented here are those for E. coli from the European Committee on Antimicrobial Susceptibility Testing (EUCAST) [13], except for nalidixic acid, sulfamethoxazole and tetracycline, for which breakpoints from the Clinical and Laboratory Standards Institute (CLSI) [14] were used.

The MIC values of $2 \mu \mathrm{g} / \mathrm{mL}$ for cefepime and for ceftazidime were above the susceptible EUCAST breakpoint (S $\leq 1 \mu \mathrm{g} / \mathrm{mL})$ and within the intermediate range (non-susceptible).

Whole genome sequencing and in silico analysis

Total DNA from OXA-48-like-positive strains was isolated using the DNeasy Blood and Tissue Kit (Qiagen, Venlo, the Netherlands), and purified using the AMPure XP paramagnetic bead-based chemistry (Beckmann Coulter, Brea, US). All CP E. coli were sequenced $(2 \times 150 \mathrm{bp}$ paired-end) using the Illumina HiSeq technology (Illumina Inc, San Diego, US) at the Eurofins Institut (Konstanz, Germany). The genomic DNA of strain AR24.2b was additionally sequenced with MinION on a R9.4 Spot On flow cell using the library preparation kit SQK-LSK108 (Oxford Nanopore Technologies (ONT), Oxford, United Kingdom (UK)). Genome assembly of all strains was performed using SPAdes (v3.12.0) and the obtained contigs were used for in silico analysis. The genome assembly of strain AR24.2b was performed by Unicycler (vo.4.4), using both long (ONT) and short reads (Illumina), as well as by CANU (v1.7) that only uses the ONT long reads. The obtained scaffolds were polished by read-mapping with paired-end Illumina reads using Pilon (v1.22) and with Geneious software v10.1.3 (Biomatters Ltd, Auckland, New Zealand). Annotation was performed with PROKKA v1.12. The paired-end reads of all CP E. coli strains were mapped to the final annotated plasmid sequences of AR24.2b. Online tools available at the Center for Genomic Epidemiology (Technical University of Denmark DTU, Lyngby, Denmark) were used to detect known ARG (ResFinder 3.0) to determine incompatibility (Inc) groups of the plasmids, (PlasmidFinder 1.3) and for multilocus sequence typing (MLST 2.0). 
Characteristics of the carbapenemase-producing Escherichia coli isolates, Switzerland, May-August 2018 ( $\mathrm{n}=24)$

\begin{tabular}{|c|c|c|c|c|}
\hline Strains & $\mathrm{ST}^{\mathrm{a}}$ & $\begin{array}{l}\text { Resistance } \\
\text { phenotype }^{\mathrm{b}}\end{array}$ & $\begin{array}{l}\text { Resistance genes }{ }^{c} \text { and } \\
\text { mutations }^{d}\end{array}$ & Localisation $^{e}$ \\
\hline \multirow{3}{*}{$\begin{array}{l}\text { AR8.2b; AR10.2b; AR17.2b; AR22.1; AR22.2; AR24.2b; } \\
\text { AR25.2; AR26.2c; AR31.2a; AR33.2a; AR44.2; AR46.2b; } \\
\text { AR62.2; AR76.2; AR76.3; AR81.2; AR94.2b; AR96.2a; } \\
\text { AR96.3; AR135.2a; AR145.2; AR148.2 }(\mathrm{n}=22)\end{array}$} & \multirow{3}{*}{410} & \multirow{3}{*}{$\begin{array}{l}\text { AMP-CIP-ETP-FEP- } \\
\text { FOT-NAL-TAZ }\end{array}$} & blaCMY-42, sugE & Plasmid Incl1, 47-kb \\
\hline & & & blaOXA-181, qnrS1 & Plasmid Inc $X_{3}, 51-k b$ \\
\hline & & & $\begin{array}{c}\text { GyrA (Ser83Leu, } \\
\text { Asp87Asn); ParC (Ser8olle) }\end{array}$ & Chromosome \\
\hline \multirow{3}{*}{ AR89.2b $(n=1)$} & \multirow{3}{*}{410} & \multirow{3}{*}{$\begin{array}{c}\text { AMP-CIP-ETP-FOT- } \\
\text { NAL }\end{array}$} & bla0XA-181, anrS1 & Plasmid Inc $X_{3}, 51-k b$ \\
\hline & & & None & Plasmid Incl1, 88-kb ${ }^{f}$ \\
\hline & & & $\begin{array}{c}\text { GyrA (Ser83Leu, } \\
\text { Asp87Asn); ParC (Ser8olle) }\end{array}$ & Chromosome \\
\hline \multirow{3}{*}{ AR144.2 $(n=1)$} & \multirow{3}{*}{410} & \multirow{3}{*}{$\begin{array}{l}\text { AMP-CIP-ETP-FOT- } \\
\text { NAL-TET }\end{array}$} & blaOXA-181, qnrS1 & Plasmid Inc $X_{3}, 51-k b$ \\
\hline & & & blaTEM-1B, tet(A) & Plasmid Incl1, 98-kb ${ }^{f}$ \\
\hline & & & $\begin{array}{c}\text { GyrA (Ser83Leu, } \\
\text { Asp87Asn); ParC (Ser8olle) }\end{array}$ & Chromosome \\
\hline
\end{tabular}

AMP: ampicillin; CHL: chloramphenicol; CIP: ciprofloxacin; COL: colistin; ETP: ertapenem; FEP: cefepime; FOT: cefotaxime; GEN: gentamicin; IMI: imipenem; MERO: meropenem; NAL: nalidixic acid; SMX: sulfamethoxazole; ST: sequence type; TAZ: ceftazidime; TET: tetracycline; TGC: tigecycline; TMP: trimethoprim.

${ }^{a}$ Sequence type based on multilocus sequence typing was determined with MLSTtool 2.0.

${ }^{\mathrm{b}}$ Results interpreted according to the EUCAST and CLSI criteria (see Table 1).

c Resistance genes were detected with ResFinder 3.0: blaCMY-42, cephalosporinase CMY-42 gene (resistance to FEP, TAZ); blaOXA-181, carbapenemase OXA-181 gene (resistance to AMP, ETP, FOT); qnrS1, DNA gyrase protection gene to fluoroquinolones (low-level resistance to fluoroquinolones); blaTEM-1b, $\beta$-lactamase TEM-1b gene (resistance to AMP); tet(A), tetracycline efflux gene (resistance to TET); sugE, quaternary ammonium compound resistance gene.

${ }^{d}$ Chromosomal mutations were detected with ResFinder 3.0. GyrA (Ser83Leu and Asp87Asn), combined with ParC (Ser8olle), mutations in the topoisomerase II and IV (high-level resistance to fluoroquinolones).

${ }^{\mathrm{e}}$ Incompatibility groups (Inc) were determined with PlasmidFinder 1.3.

${ }^{\mathrm{f}}$ As determined using plasmid SPAdes (v3.12.0).

Transposable elements present in the plasmids were identified using ISfinder (https://isfinder.biotoul.fr/). Blast ring image generator (BRIG) was used to generate circular map comparisons of plasmids (based on BLASTn) [15]. Comparison with closely related plasmids from the National Center for Biotechnology Information (NCBI) was achieved using the NCBI tool BLASTn (https://blast.ncbi.nlm.nih.gov/Blast.cgi, accessed November 2018).

\section{Phylogenetic analyses}

An ad hoc core genome multilocus sequence typing (cgMLST) scheme was created using SeqSphere+(v. 401, Rindom GmbH, Münster, Germany). The E. coli of sequence type (ST) 410 from a dog in the UK (GenBank accession number (P031653) was defined as the reference genome from which 4,177 coding regions of genes were extracted by the cgMLST Target Finder v1.4. The contigs obtained from SPAdes of all 24 CP E. coli in this study, together with the reference genome and with six other genomes of $E$. coli ST410 isolated from humans or from the environment and published in GenBank (GenBank accession numbers CP029630, CP027205, CP026473, CP024801, CP023899 and CP018965), were loaded and searched for the 4,177 gene targets using built-in BLAST. Overall, 3,778 genes were shared among all isolates, which we defined as the core genome. The visualisation of the phylogenetic distance was achieved using the allelic profile of all strains to generate a neighbour-joining tree using SeqSphere+with the parameters 'pairwise ignoring missing values; \% columns difference' for the distance calculation.

\section{Ethical statement}

The study protocol was approved by the national ethics committee (BE 16/18) and signed informed consent was obtained from the owners before enrolment of the animals in the study.

\section{Results}

\section{Occurrence of carbapenemase- producing Escherichia coli}

Rectal swabs were taken from 100 dogs and 34 cats at admission to a large veterinary referral clinic in Switzerland between 1 May and 31 August 2018. Carriage of CP E. coli was detected in only one dog and none of the cats at admission ( $0.75 \%$; $95 \%$ confidence interval (CI): 0-2.2).

In contrast, 17 of 76 dogs and four of 21 cats that were resampled at discharge were found to harbour CP E. coli (21.6\%; 95\% C: $13.4-29.8)$ indicating nosocomial acquisition of the bacteria. The 37 remaining animals were not available for resampling $(n=22)$ or deceased $(n=15)$. 


\section{FIGURE 3}

Circular maps of the resistance plasmids pAN-OXA-181 and pAN-CMY-42, Switzerland, May-August 2018 ( $\mathrm{n}=2$ )

A.

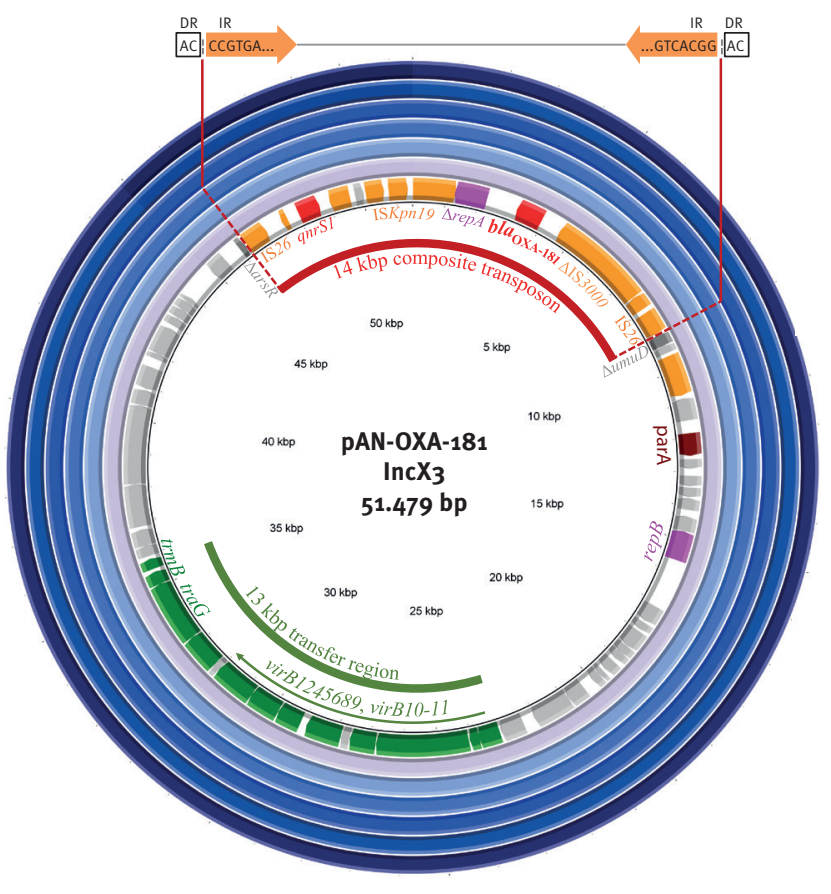

1. $\mathrm{PAMA1167-OXA-181}$

$100 \%$ Identity

$95 \%$ Identity

$90 \%$ Identity

3. $\mathrm{pKS} 22-\mathrm{OXA}-181$

$100 \%$ Identity

$95 \%$ Identity

- $90 \%$ Identity

5. pSTIB

$100 \%$ Identity

95\% Identity

90\% Identity
2. POXA181_14828

$100 \%$ Identity

- $95 \%$ Identity

- $90 \%$ Identity

4. pEc31346-OXA-181

$100 \%$ Identity

$95 \%$ Identity

$90 \%$ Identity

6. $\mathrm{pEC} 21-0 \mathrm{XA}-181$

$100 \%$ Identity

$95 \%$ Identity

90\% Identity
B.

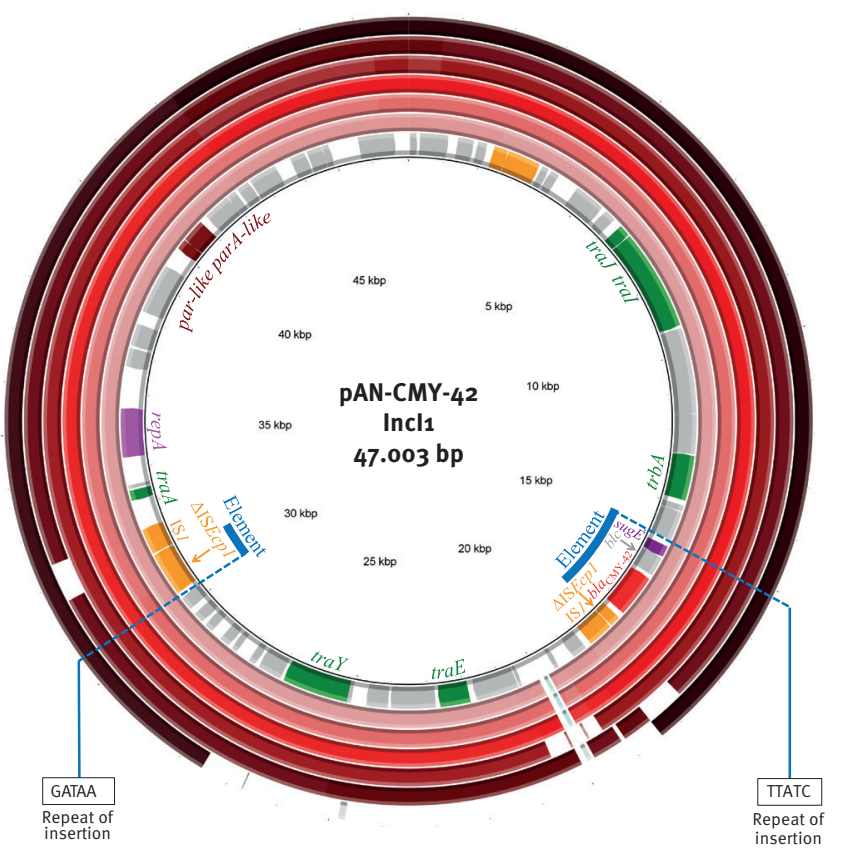

1. $\mathrm{pCMY} 42 \_M 217$

2. $\mathrm{pCMY42 \_ AR \_ 0137}$

$100 \%$ Identity

$95 \%$ Identity

- $90 \%$ Identity

$100 \%$ Identity

95\% Identity

90\% Identity

3. pCARB35_03

4. $\mathrm{pCMY}$ 42_WCHEC13-8

$100 \%$ Identity

$95 \%$ Identity

- $90 \%$ Identity

100\% Identity
$95 \%$ Identity
$90 \%$ Identity

5. pV233-b

$100 \%$ Identity

- $95 \%$ Identity

90\% Identity
6. pIBAC_Incl1_CMY-42

$100 \%$ Identity

95\% Identity

90\% Identity

The coloured outer rings indicate regions of homology of the pAN-OXA-181 (GenBank accession number MK416154) and pAN-CMY-42 (GenBank acc. no. MK416155) plasmid of strain AR24.2b with other plasmids, based on BLASTn.

Left panel: pAN-OXA-181. The rings from the inner to the outer represent plasmids pAMA1167-0XA-181 (GenBank acc. no. CP024806; 99.99\% identity, 1 single nucleotide polymorphism (SNP)), pOXA181_14828 (GenBank acc. no. KP400525; 100\% identity), pKS22-OXA-181 (GenBank acc. no. KTo05457; 99.98\%, 3 SNP), pEc31346-OXA-181 (GenBank acc. no. KX894452; 99.99\% identity, 1 SNP), pSTIB (GenBank acc. no. MG570092; 99.99\% identity, 1 SNP) and pEC21-OXA-181 (GenBank acc. no. MG893567; $99.99 \%$ identity, 1 SNP).

Left panel: pAN-CMY-42. The rings from the inner to the outer represent plasmids pCMY42_M217 (GenBank acc. no. APo19190; $99 \%$ identity, 65\% coverage), pCMY42_AR_0137 (GenBank acc. no. CP021882; 100\% identity, 68\% coverage), pCARB35_03 (GenBank acc. no. CP031656; $100 \%$ identity, 79\% coverage), pCMY42 WCHEC13-8 (GenBank acc. no. KP789019; 100\% identity, 71\% coverage), pV233-b (GenBank acc. no. LC056425; $99 \%$ identity, 46\% coverage) and pIBAC_Incl1_CMY-42 (GenBank acc. no. KY463221; $100 \%$ identity, $78 \%$ coverage).

The scale circle shows the coordinates in kbp of each plasmid. Genes are portrayed as coloured blocks in the inner ring of each circular map. Genes are coloured according to their classification. Red: antimicrobial resistance genes; dark purple: surface disinfectants resistance genes; orange: transposases; fuchsia: genes implicated in replication; green: transfer-associated genes; brown: partition genes; grey: other genes. The $\triangle$ umud and $\triangle a r S R$ genes are coloured in darker grey, flanking the composite transposon of pAN-OXA-181. The blc gene of pAN-CMY-42 is indicated in the map to better represent the composition of the native $4 \mathrm{~kb}$ element. 
The dog positive for CP E. coli at admission was not under antimicrobial treatment at arrival and had no previous history of hospitalisation at this clinic, but whether it was previously hospitalised in another clinic is not known. Nor was it the first animal tested positive during the study period. This dog was still positive at discharge (Figure 1).

The demographics, hospitalisation details and antimicrobial treatments of the CP E. coli-carrying animals are summarised in Table 1. Animals were presented to the emergency service of the hospital with a wide range of complaints. All cats and 13 of the 17 dogs spent time in the intensive care unit of the hospital. The majority of dogs (14/17) and cats (3/4) were treated with antimicrobials during their hospitalisation; however, none of them received carbapenems.

Follow-up samples were obtained within 36 to 101 days after discharge from 12 of 21 CP E. coli-positive animals. The other nine animals could not be retested because they had died $(n=3)$, or because the owners withdrew their participation in the study $(n=6)$. Two cats still carried CP E. coli 36 days and 99 days after discharge (Figure 1). Seven of the 12 animals that underwent a first follow-up could be retested for a second time within a time interval of 109 to 166 days after discharge of the clinic, including the two cats that were still positive after the first follow-up. None of them were found to be shedding CP E. coli at the second follow-up (Figure 1).

\section{Phylogenetic analyses}

All $24 \mathrm{CP}$ E. coli detected in this study belonged to ST410. Analysis of the 24 strains using cgMLST confirmed that they were all highly related, all clustering in the same branch of the phylogenetic tree, in contrast to the sequences of seven other and independent $E$. coli ST410 from animal, environmental and human origin. The most closely related strain was a canine $E$. coli isolated in the UK in 2018, which carried the carbapenemase gene bla NDM-5, the plasmid-mediated AmpC (pAmpC) cephalosporinase gene bla ${ }_{\text {сMY-42 }}$, and the $\beta$-lactamase gene bla ${ }_{\text {TEM-19o }}$ [16] (GenBank accession number (Po31653) (Figure 2).

\section{Antimicrobial resistance profile of the} carbapenemase-producing $E$. coli

All CP E. coli exhibited the same antimicrobial resistance profile; they were non-susceptible to ampicillin, cefepime, cefotaxime, ceftazidime, ciprofloxacin, ertapenem and nalidixic acid, except for one isolate (AR144.2) which was susceptible to ceftazidime and resistant to tetracycline (Table 2 ).

All isolates carried the carbapenemase gene bla ${ }_{\text {OXA-181, }}$, which has been associated with a low level of resistance to carbapenems [4,17]. The isolates had MICs below the resistance breakpoints set by EUCAST for imipenem and meropenem but were resistant to ertapenem, with MICs above the resistance breakpoint
(Table 2). All isolates also contained the qnrS1 gene encoding a DNA gyrase protection protein which confers low-level resistance to fluoroquinolones, as well as a serine to leucine substitution at position 83 and an aspartic acid to asparagine substitution at position 87 in GyrA, and a serine to isoleucine substitution at position 80 in ParC. These amino acid substitutions are known to confer high-level resistance to fluoroquinolones in E. coli [1].

The pAmpC cephalosporinase bla ${ }_{\text {сMY-42 }}$ was also found in all isolates, except in the two isolates (AR89.2b and AR144.2) which were not resistant to cefepime and ceftazidime. The tetracycline resistance found only in AR144.2 was associated with the tetracycline efflux gene tet(A). This isolate was also the only one that contained the bla ${ }_{\mathrm{TEM}-\mathrm{B}} \beta$-lactamase gene (Table 3).

\section{Characterisation of the plasmids containing resistance genes}

The bla ${ }_{\text {OXA-181 }}$ and qnrS1 genes were co-localised on a $51 \mathrm{~kb}$ IncX3 plasmid (pAN-OXA-181) in strain AR24.2b (Figure 3). Plasmid mapping showed that all other 23 isolates also harboured this same plasmid. Plasmid pAN-OXA-181 was $>99.9 \%$ similar to other Inc $X_{3}$ plasmids from human $E$. coli strains in China (pOXA181_14828 and pEC21-OXA-181 [18,19], Denmark (pAMA1167-OXA-181) [20], and Lebanon (pSTIB) [21], as well as from a porcine $E$. coli strain in Germany (pEc31346-OXA-181) [22]. Plasmid pAN-OXA-181 differed by 3 SNPs from pKS22-OXA-181 which was detected in a Klebsiella variicola isolated from fresh vegetables imported into Switzerland [23] (Figure 3).

Both bla ${ }_{\text {OXA-181 }}$ and $q n r S_{1}$ were found within a composite transposon in pAN-OXA-181, which was integrated between $u m u D$ and $a s r R$, similarly to pOXA-181_14828 [18]. The composite transposon was flanked by two copies of the insertion sequence IS26 which were oriented in the same direction. Two $50 \mathrm{bp}$ inverted repeats IRL and IRR, each preceded by a 2 bp AC direct repeat, were found at both ends of the IS26-flanked composite transposon, which is indicative of a transposition event [19]. Plasmid pAN-OXA-181 also contained the replication gene rep $B$ and a $23 \mathrm{~kb}$ transfer region containing the virB1-2-4-5-6-8-9-10-11, traG and $\operatorname{trm} B$ genes (Figure 3).

Strain AR24.2b also contained a $47 \mathrm{~kb}$ Incl1 plasmid (pAN-CMY-42) which harboured the pAmpC gene bla ${ }_{\mathrm{CMY}-42}$ (Figure 3). The bla ${ }_{\mathrm{CMY}-42}$ gene is commonly found in plasmids within a $4 \mathrm{~kb}$ element composed by ISEcP1, an outer membrane lipoprotein gene blc and a quaternary ammonium compound (QAC) resistance gene sugE (ISEcp1 - bla ${ }_{\text {сMY-42 }}$ - blc - sugE) [24]. In pANCMY-42 however, $\triangle I S E C P 1$ of this element was split by the insertion of a copy of IS1, and subsequent homologous recombination with another copy of $\mathrm{IS}_{1}$ led to its modified structure, as previously described [24]. This recombination is supported by the inversion of the insertion repeats (GATAA and TTATC) (Figure 3). 


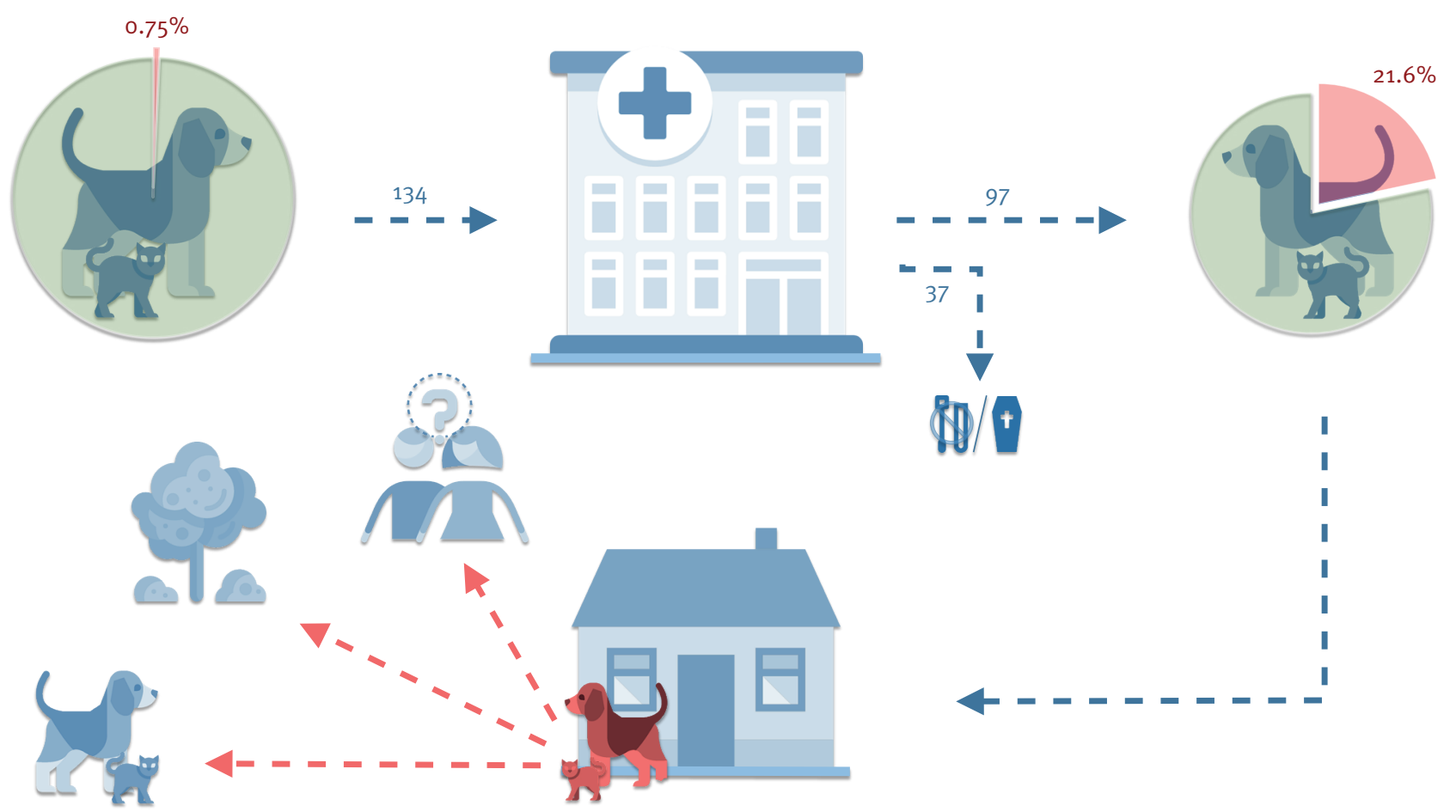

Plasmid pAN-CMY-42 also contains the replication gene repA and transfer genes traA, traE, tral, tral, traY and trbA.

Plasmid pAN-CMY-42 differed from other CMY-42containing Incl1 plasmids by its size and structure. The most closely related Incl1 CMY-42 plasmids deposited so far in GenBank were larger than pAN-CMY-42 by $1-50 \mathrm{~kb}$, including plasmids isolated from canine $E$. coli in the UK (pCARB35_03) [16], human patients in Myanmar (pCMY42_M217) [25], China (pCMY42_ WCHEC13-8) [24] and Italy (pIBAC_Incl1_CMY-42) [26], as well as from $E$. coli isolated from the environment (pV233-b) [27], and from an unknown source (pCMY42_ AR_0137 (Figure 3).

Plasmid mapping of the other $23 E$. coli showed that 21 isolates also contained this same plasmid (pANCMY-42), whereas the two isolates AR89.2 and AR144.2 lacked the $4 \mathrm{~kb}$ element (ISECP1 - bla ${ }_{\mathrm{CMY}-42}$ - blc sugE) on their Incli plasmid.

Plasmids pAN-OXA-181, pAN-CMY-42 and the chromosome of $E$. coli strain AR24.2b have been deposited in GenBank under accession numbers MK416154, MK416155 and CP035944, respectively.

\section{Discussion}

Surveillance of enteral carriage of antimicrobial-resistant bacteria in dogs and cats before and after hospitalisation in a companion animal hospital in Switzerland revealed the potential role of small animal clinics as an underestimated hotspot for acquisition of CP E. coli. In the particular situation presented here, almost one quarter of the hospitalised animals acquired a specific carbapenem-, cephalosporin- and fluoroquinoloneresistant clone of $\mathrm{ST}_{410}$ within a time span of 1-9 days of hospitalisation. The only dog found to be carrier of this clone at admission was chronologically not the first to be found positive during the study period, indicating that it was not the primary source of this strain. It may, however, have contributed to further enhance dissemination of the clone into the hospital. Long-term carriage beyond 108 days was only documented in one cat among the 10 dogs and two cats that were followed up. Nevertheless, all 21 animals that returned home with $C P$ E. coli posed a potential risk of disseminating hospital-acquired CP E. coli into the environment and possibly to other animals and humans (Figure 4). Although the risk of CP Enterobacteriaceae transmission may be related to their relative abundance in faeces, our study was not set up to quantify the effective number of CP Enterobacteriaceae in faeces because it used enrichment and selective plates.

This figure shows the results of the screening of 134 animals at admission to the veterinary hospital and of 97 animals at discharge. It illustrates the potential risk for the environment, humans and other companion animals with regard to the carriage of CP E. coli by animals that have returned to their homes after having acquired CP E. coli during hospitalisation. 
It is important to extend screening and detection methods in veterinary settings and diagnostic laboratories to detect such bacteria of medical and public health importance [4]. Detection of carbapenemases of the OXA-48 family may be challenging because of their low hydrolysing activity which specifies low MIC to carbapenems $[4,5]$. Application of low MIC screening values would allow identifying the OXA-181-producing $E$. coli isolates in our study since they exhibited MICs higher than the resistance breakpoint of $>0.5 \mu \mathrm{g} / \mathrm{mL}$ for ertapenem and higher than the meropenem screening cut-off value of $>0.125 \mu \mathrm{g} / \mathrm{mL}$ recommended by EUCAST [13].

Phylogenetic analyses of the CP E. coli using 3,778 genes of the core genome (cgMLST) confirmed high genetic relationship shared among all the $E$. coli isolates of ST410 from this study. This sequence type has recently been reported as a new emerging international highrisk clone, non-susceptible to critically important antibiotics such as fluoroquinolones, third-generation cephalosporins and carbapenems and with the potential of cross-sectorial transmission between wildlife, humans, pets and the environment $[28,29]$. Escherichia coli ST410 harbouring bla ${ }_{\text {oxA-181 }}$ was first described in human infections in China in 2015 [18], then in Denmark in 2017 [20] and in Italy in 2018 [30], but so far it has not been reported in humans in Switzerland. These previously described human E. coli ST410 producing OXA-181 clustered into other branches of the cgMLST phylogenetic tree than the strain isolated in our study, indicating an independent origin. Another CP E. coli ST410 was identified in a dog in the UK in 2018 [16], GenBank accession number CP031653), emphasising the potential of ST410 for dissemination in the veterinary setting. This canine $E$. coli ST410 from the UK exhibited a slightly different cgMLST clustering and contained NDM-5 as carbapenemase rather than OXA181. Although the two canine E. coli ST410 from the UK and Switzerland contained CMY-42 Incl1 plasmids which were highly similar, the one from Switzerland was lacking a $12 \mathrm{~kb}$ region compared with the CMY-42 Incl1 plasmid of the canine $E$. coli from the UK.

The present study revealed for the first time an OXA-181producing $E$. coli ST410 associated with hospitalisation of companion animals. Carriage of carbapenemaseproducing bacteria has so far not been reported among animals in Switzerland, but one study already reported OXA-181 in $K$. variicola isolated from imported fresh vegetables [23]. The presence of the same plasmid containing bla ${ }_{\text {OXA-181 }}$ (pAN-OXA-181) in Enterobacteriaceae from companion animals, humans and vegetables suggest that interspecies transmission of this plasmid between $E$. coli and other Enterobacteriaceae is very likely. Nevertheless, the origin of the $E$. coli ST410 containing the carbapenemase plasmid pAN-OXA-181 in the veterinary setting is intriguing. Carbapenems are not used in this clinic and are therefore not likely to be the driving force for the selection and maintenance of this carbapenem-resistant $E$. coli ST410. The use of fluoroquinolones and $\beta$-lactams (Table 1 ) may have contributed to the selection of this ST410 clone or its plasmids, since the isolates also exhibited resistance to these classes of antimicrobials. That more animals were carriers of the CP ST410 clone at discharge than at admission as well as the high genetic relatedness of all isolates (including the identical plasmids) strongly indicate a common source of contamination within the hospital. Nevertheless, no cases of infection caused by an OXA-181-producing $E$. coli were revealed in dogs and cats during the 7 months following the outbreak using the same selective agar plates as those used in this study at the diagnostic unit of our institute. Of note, this referral hospital faced several cases of infections caused by a nosocomial clone of third-generationcephalosporin-resistant $K$. pneumoniae ST11 producing the pAmpC DHA in 2010-2013 [31].

As an infection control measure, thorough disinfection of the hospital environment was performed after this outbreak in 2018 using disinfectant without QAC since ST410 also contained a QAC resistance gene (sugE) on the cephalosporinase plasmid pAN-CMY-42. Furthermore, stringent hospital hygiene and environmental cleaning protocols were introduced and a staff screening campaign was initiated. Staff training was also intensified to improve hand and environmental hygiene. The results and the impact of these measures are still being analysed and will be part of a follow-up study. Although no conclusion can be drawn at this stage of the study on the impact of possible colonisation of the staff or the pet owners, the results indicate that companion animals probably disseminated CP Enterobacteriaceae via faeces in the environment after hospitalisation and may represent a potential risk for transmission of CP Enterobacteriaceae to other animals and humans in the community and a serious One Health concern.

This outbreak stresses the need for national routine monitoring of carbapenemase-producing bacteria in companion animals using a representative sampling strategy analogous to the national surveillance of antibiotic resistance in food-producing animals at slaughterhouses in Switzerland and the European Union [32]. Based on the results of our study, we strongly recommend monitoring hospitalised companion animals for CP Enterobacteriaceae carriage at discharge using a sampling strategy representative of the number of hospitalised animals in the enrolled clinics. Such a surveillance programme should identify high-risk companion animal clinics and improve infection prevention and control as well as hygiene standards.

\section{Conclusion}

This study provided evidences that dogs and cats can acquire CP Enterobacteriaceae within a short time of hospitalisation. Even if the colonisation of the animals did not last longer than 40 days in the majority of the animals, shedding of CP Enterobacteriaceae after 
hospitalisation represents a public health and environmental concern. Early detection and nationwide surveillance of carbapenemase-producing isolates, as well as effective infection prevention and control measures, need to be implemented in veterinary settings to limit the spread of high-risk clones in animals, humans and the environment.

\section{Acknowledgements}

This study was financed by the Swiss Federal Food Safety and Veterinary Office [FSVO Grant no. 1.18.10 "Prevalence and identification of critical points for transmission of multidrug-resistant bacteria in small animal clinics - towards evidence-based guidelines for infection prevention and control" to SS, BW, SGB, AE and VP], and by the Swiss National Science Foundation [SNSF grant no. 177378 within the National Research Programme NRP72 "Antimicrobial Resistance" to AE and VP]. We are grateful to all participating owners who kindly made the sampling of their animals possible.

\section{Conflict of interest}

None declared.

\section{Authors' contributions}

$\mathrm{SS}, \mathrm{BW}, \mathrm{SGB}, \mathrm{AE}$ and $\mathrm{VP}$ contributed to the design of the study. VD sampled the animals. AN and AC isolated and identified the strains. SS and VD collected and analyzed the clinical data. AN and MB performed experiments and genomic analysis. $M B$ and $M C$ performed Minlon sequencing. AN, MB and VP interpreted bacteriological and molecular the data, and AN and VP wrote the manuscript. All authors reviewed the manuscript.

\section{References}

1. Hooper DC, Jacoby GA. Mechanisms of drug resistance: quinolone resistance. Ann N Y Acad Sci. 2015;1354(1):12-31. https://doi.org/10.1111/nyas.12830 PMID: 26190223

2. Fernandes R, Amador P, Prudêncio C. $\beta$-lactams: chemical structure, mode of action and mechanisms of resistance. Rev Med Microbiol. 2013;24(1):7-17. https://doi.org/10.1097/ MRM.ob013e3283587727

3. Patel G, Bonomo RA. "Stormy waters ahead": global emergence of carbapenemases. Front Microbiol. 2013;4:48. https://doi.org/10.3389/fmicb.2013.00048 PMID: 23504089

4. Nordmann P, Gniadkowski M, Giske CG, Poirel L, Woodford $\mathrm{N}$, Miriagou $\mathrm{V}$, et al. Identification and screening of carbapenemase-producing Enterobacteriaceae. Clin Microbiol Infect. 2012;18(5):432-8. https://doi.org/10.1111/j.14690691.2012.03815.x PMID: 22507110

5. Poirel L, Potron A, Nordmann P. OXA-48-like carbapenemases: the phantom menace. J Antimicrob Chemother. 2012;67(7):1597606. https://doi.org/10.1093/jac/dks121 PMID: 22499996

6. Poirel L, Stephan R, Perreten V, Nordmann P. The carbapenemase threat in the animal world: the wrong culprit. J Antimicrob Chemother. 2014;69(7):2007-8. https://doi. org/10.1093/jac/dku054 PMID: 24576948

7. Stolle I, Prenger-Berninghoff E, Stamm I, Scheufen S, Hassdenteufel E, Guenther S, et al. Emergence of OXA 48 carbapenemase-producing Escherichia coli and Klebsiella pneumoniae in dogs. J Antimicrob Chemother. 2013;68(12):2802-8. https://doi.org/10.1093/jac/dkt259 PMID: 23833179

8. Melo LC, Boisson MN, Saras E, Médaille C, Boulouis HJ, Madec JY, et al. OXA-48-producing ST372 Escherichia coli in a French dog. J Antimicrob Chemother. 2017;72(4):1256-8. PMID: 28039279
9. Liu X, Thungrat K, Boothe DM. Occurrence of OXA-48 carbapenemase and other beta-lactamase genes in ESBLproducing multidrug resistant Escherichia coli from dogs and cats in the United States, 2009-2013. Front Microbiol. 2016;7:1057. PMID: 27462301

10. Yousfi M, Touati A, Mairi A, Brasme L, Gharout-Sait A, Guillard $\mathrm{T}$, et al. Emergence of carbapenemase-producing Escherichia coli isolated from companion animals in Algeria. Microb Drug Resist. 2016;22(4):342-6. https://doi.org/10.1089/ mdr.2015.0196 PMID: 26741510

11. Gentilini F, Turba ME, Pasquali F, Mion D, Romagnoli N, Zambon E, et al. Hospitalized pets as a source of carbapenemresistance. Front Microbiol. 2018;9(2872):2872. https://doi. org/10.3389/fmicb.2018.02872 PMID: 30574124

12. Pires J, Novais A, Peixe L. Blue-carba, an easy biochemical test for detection of diverse carbapenemase producers directly from bacterial cultures. J Clin Microbiol. 2013;51(12):4281-3. https://doi.org/10.1128/JCM.01634-13 PMID: 24108615

13. The European Committee on Antimicrobial Susceptibility Testing (EUCAST). Breakpoint tables for interpretation of MICS and zone diameters. Version 9.0, 2019. Växjö: EUCAST; 2019. Available from: http://www.eucast.org/fileadmin/src/media/ PDFs/EUCAST_files/Breakpoint_tables/v_9.0_Breakpoint_ Tables.pdforg

14. Clinical and Laboratory Standards Institute (CLSI). Performance standards for antimicrobial susceptibility testing. 26th ed. CLSI document M100-S27. Wayne, PA: CLSI; 2017.

15. Alikhan NF, Petty NK, Ben Zakour NL, Beatson SA. BLAST Ring Image Generator (BRIG): simple prokaryote genome comparisons. BMC Genomics. 2011;12(1):402. https://doi. org/10.1186/1471-2164-12-402 PMID: 21824423

16. Reynolds ME, Phan HTT, George S, Hubbard ATM, Stoesser $\mathrm{N}$, Maciuca IE, et al. Occurrence and characterization of Escherichia coli ST410 co-harbouring blaNDM-5, blaCMY-42 and blaTEM-190 in a dog from the UK. J Antimicrob Chemother. 2019;74(5):1207-11. https://doi.org/10.1093/jac/dkzo17 PMID: 30753576

17. Evans BA, Amyes SG. OXA $\beta$-lactamases. Clin Microbiol Rev. 2014;27(2):241-63. https://doi.org/10.1128/CMR.00117-13 PMID: 24696435

18. Liu Y, Feng Y, Wu W, Xie Y, Wang X, Zhang X, et al. First report of OXA-181-producing Escherichia coli in China and characterization of the isolate using whole-genome sequencing. Antimicrob Agents Chemother. 2015;59(8):5022-5. https://doi.org/10.1128/AAC.00442-15 PMID: 26014927

19. Qin S, Cheng J, Wang P, Feng X, Liu HM. Early emergence of OXA-181-producing Escherichia coli ST410 in China. J Glob Antimicrob Resist. 2018;15:215-8. https://doi.org/10.1016/j. jgar.2018.06.017 PMID: 30393155

20. Overballe-Petersen S, Roer L, Ng K, Hansen F, Justesen US, Andersen LP, et al. Complete nucleotide sequence of an Escherichia coli sequence type 410 strain carrying blaNDM-5 on an IncF multidrug resistance plasmid and blaOXA-181 on an Inc X3 plasmid. Genome Announc. 2018;6(5):e01542-17. https://doi.org/10.1128/genomeA.01542-17 PMID: 29437102

21. Bitar I, Dagher C, Salloum T, Araj G, Tokajian S. First report of an Escherichia coli from Lebanon carrying an OXA-181 carbapenemase resistance determinant. J Glob Antimicrob Resist. 2018;12:113-4. https://doi.org/10.1016/j. jgar.2018.01.002 PMID: 29353076

22. Pulss S, Semmler T, Prenger-Berninghoff E, Bauerfeind $R$, Ewers C. First report of an Escherichia coli strain from swine carrying an OXA-181 carbapenemase and the colistin resistance determinant MCR-1. Int I Antimicrob Agents. 2017;50(2):232-6. https://doi.org/10.1016/j. ijantimicag.2017.03.014 PMID: 28666753

23. Zurfluh K, Poirel L, Nordmann P, Klumpp J, Stephan R. First detection of Klebsiella variicola producing OXA-181 carbapenemase in fresh vegetable imported from Asia to Switzerland. Antimicrob Resist Infect Control. 2015;4(1):38. https://doi.org/10.1186/s13756-015-0080-5 PMID: 26448862

24. Feng Y, Yang P, Xie Y, Wang X, McNally A, Zong Z. Escherichia coli of sequence type 3835 carrying blaNDM-1, blaCTX-M-15, blaCMY-42 and blaSHV-12. Sci Rep. 2015;5(1):12275. https:// doi.org/10.1038/srep12275 PMID: 26194736

25. Sugawara Y, Akeda Y, Sakamoto N, Takeuchi D, Motooka D, Nakamura S, et al. Genetic characterization of blaNDMharboring plasmids in carbapenem-resistant Escherichia coli from Myanmar. PLoS One. 2017;12(9):e0184720. https://doi. org/10.1371/journal.pone.0184720 PMID: 28910381

26. Bitar I, Piazza A, Gaiarsa S, Villa L, Pedroni P, Oliva E, et al. ST405 NDM-5 producing Escherichia coli in Northern Italy: the first two clinical cases. Clin Microbiol Infect. 2017;23(7):48990. https://doi.org/10.1016/j.cmi.2017.01.020 PMID: 28159670

27. Akiba M, Sekizuka T, Yamashita A, Kuroda M, Fujii Y, Murata M, et al. Distribution and relationships of antimicrobial resistance 
determinants among extended-spectrum-cephalosporinresistant or carbapenem-resistant Escherichia coli isolates from rivers and sewage treatment plants in India. Antimicrob Agents Chemother. 2016;60(5):2972-80. https://doi. org/10.1128/AAC.01950-15 PMID: 26953207

28. Roer L, Overballe-Petersen S, Hansen F, Schønning K, Wang M, Røder BL, et al. Escherichia coli sequence type 410 is causing new international high-risk clones. MSphere. 2018;3(4):e00337-18. https://doi.org/10.1128/ mSphere.00337-18 PMID: 30021879

29. Schaufler K, Semmler T, Wieler LH, Wöhrmann M, Baddam R, Ahmed $\mathrm{N}$, et al. Clonal spread and interspecies transmission of clinically relevant ESBL-producing Escherichia coli of ST410-another successful pandemic clone? FEMS Microbiol Ecol. 2016;92(1):fiv155. https://doi.org/10.1093/femsec/fiv155 PMID: 26656065

30. Piazza A, Comandatore F, Romeri F, Pagani C, Floriano AM, Ridolfo A, et al. First report of an ST410 OXA-181 and CTX-M-15 coproducing Escherichia coli clone in Italy: a wholegenome sequence characterization. Microb Drug Resist. 2018;24(8):1207-9. https://doi.org/10.1089/mdr.2017.0366 PMID: 29473791

31. Wohlwend N, Endimiani A, Francey T, Perreten V. Thirdgeneration-cephalosporin-resistant Klebsiella pneumoniae isolates from humans and companion animals in Switzerland: spread of a DHA-producing sequence type 11 clone in a veterinary setting. Antimicrob Agents Chemother. 2015;59(5):2949-55. https://doi.org/10.1128/AAC.04408-14 PMID: 25733505

32. Federal Office of Public Health (FOPH) and Federal Food Safety and Veterinary Office. Swiss Antibiotic Resistance Report 2018. Usage of Antibiotics and Occurrence of Antibiotic Resistance in Bacteria from Humans and Animals in Switzerland. Novembe 2018; FOPH publication number: 2018-OEG-87. Bern: FOPH; 2018. Available from: https://www.bag.admin.ch/dam/bag/de/ dokumente/mt/star/swiss-antibiotic-resistance-report-2018. pdf

\section{License, supplementary material and copyright}

This is an open-access article distributed under the terms of the Creative Commons Attribution (CC BY 4.0) Licence. You may share and adapt the material, but must give appropriate credit to the source, provide a link to the licence and indicate if changes were made.

Any supplementary material referenced in the article can be found in the online version.

This article is copyright of the authors or their affiliated institutions, 2019. 